\title{
Effects of Prenatal Alcohol Exposure on the Volumes of the Lateral and Medial Walls of the Intraparietal Sulcus
}

\begin{abstract}
Marlie Miles ${ }^{1,2,3 *}$, Fleur L. Warton ${ }^{1,2,3}$, Ernesta M. Meintjes ${ }^{1,2,3,4 *}$, Christopher D. Molteno ${ }^{5}$, Joseph L. Jacobson ${ }^{1,5,6}$, Sandra W. Jacobson ${ }^{1,5,6}$ and Christopher M. R. Warton ${ }^{1}$

${ }^{1}$ Department of Human Biology, Faculty of Health Sciences, University of Cape Town, Cape Town, South Africa, ${ }^{2}$ Biomedical Engineering Research Centre, Division of Biomedical Engineering, Faculty of Health Sciences, University of Cape Town, Cape Town, South Africa, ${ }^{3}$ Neuroscience Institute, University of Cape Town, Cape Town, South Africa, ${ }^{4}$ Cape Universities Body Imaging Centre, University of Cape Town, Cape Town, South Africa, ${ }^{5}$ Department of Psychiatry and Mental Health, Faculty of Health Sciences, University of Cape Town, Cape Town, South Africa, ${ }^{6}$ Department of Psychiatry and Behavioral Neurosciences, Wayne State University School of Medicine, Detroit, MI, United States
\end{abstract}

Fetal alcohol spectrum disorders (FASD) continue to be the leading preventable cause of intellectual disability in the U.S., Europe, and in endemic areas, such as the Western Cape region of South Africa. Arithmetic is highly sensitive to prenatal alcohol exposure (PAE). The intraparietal sulcus (IPS) is known to play a critical role in number processing. In this study, we investigate whether smaller IPS volumes play a role in the numberprocessing deficits observed in children with PAE. Participants were 52 9- to 14year-old children from a historically disadvantaged community in Cape Town, who are participating in our ongoing studies on the effects of PAE on the brain. The IPS was manually parcellated into its medial (MIPS) and lateral (LIPS) walls on magnetic resonance images. The study aimed to examine: (1) the effects of PAE on IPS regional volumes and asymmetry, (2) whether IPS regional volumes are related to number processing performance and, if so, whether these relations are moderated by PAE and (3) potential mediation by regional IPS volumes of the relation between PAE and number processing performance. Total intracranial volume (TIV) was associated with volumes in all regions except the right LIPS. Both left MIPS and left LIPS volumes were significantly smaller in children in the fetal alcohol syndrome (FAS)/partial FAS (PFAS) group compared to controls. The finding in the left LIPS remained significant after controlling for potential confounders and after adjustment for the smaller overall brain size of the children in the FAS/PFAS group. Smaller left LIPS volumes in the FAS/PFAS group may account for the absence of left-right asymmetry in the LIPS in children with FAS/PFAS compared to controls and nonsyndromal heavily exposed $(\mathrm{HE})$ children. Bilaterally, larger MIPS volumes were associated with better WISC IQ Arithmetic scores. These effects, however, were not moderated by the degree of PAE, and regional IPS volumes did not mediate the effect of PAE on WISC Arithmetic scores. Although we found that certain regions of the IPS were smaller in children with FAS and PFAS, these PAE-induced changes in IPS volume did not mediate the alcohol-related deficits in arithmetic.

Keywords: intraparietal sulcus (IPS), fetal alcohol spectrum disorder (FASD), arithmetic, magnetic resonance imaging (MRI), cortical volumes 


\section{INTRODUCTION}

Prenatal alcohol exposure (PAE) can lead to a range of structural and functional consequences including delayed growth, atypical facies, and intellectual disability (Jones and Smith, 1973; Hoyme et al., 2005). Fetal alcohol spectrum disorders (FASD) encompass the range of disorders that arise as a result of PAE (Bertrand et al., 2005; Hoyme et al., 2005, 2016). Fetal alcohol syndrome (FAS), the most severe form of FASD, is characterized by a distinctive pattern of craniofacial dysmorphic features (including small palpebral fissures, thin upper lip, and flat philtrum), small head circumference, and delayed pre-and/or postnatal growth, which appears to evolve with age, except in the most severely affected individuals (Jacobson et al., 2021). A diagnosis of partial FAS (PFAS) requires the presence of at least two of the sentinel facial features as well as either small head circumference, delayed growth or neurobehavioral impairment, and confirmed maternal drinking during pregnancy. A large proportion of alcohol-exposed children may, however, lack the characteristic pattern of dysmorphic facial features but exhibit the cognitive and/or behavioral deficits associated with PAE (Hoyme et al., 2005). Our study was conducted in Cape Town, South Africa, where certain historically disadvantaged communities have been reported to have amongst the highest rates of FAS in the world (Croxford and Viljoen, 1999; May et al., 2000, 2013, 2016; Jacobson et al., 2006).

Imaging studies have consistently provided evidence of cortical volume alterations in children prenatally exposed to alcohol (Rajaprakash et al., 2014; Migliorini et al., 2015). In particular, the volume (Archibald et al., 2001; Lebel et al., 2012; Meintjes et al., 2014; Rajaprakash et al., 2014) and function (Woods et al., 2015, 2017; Infante et al., 2017) of the parietal cortex are frequently implicated. Morphometric findings have, however, been inconsistent, with some studies reporting gray matter reductions (Archibald et al., 2001) and others increases (Sowell et al., 2001). Number processing, which is one of the most sensitive neurocognitive deficits associated with PAE (Streissguth et al., 1989, 1994; Kopera-Frye et al., 1996; Burden et al., 2005; Howell et al., 2006; Jacobson et al., 2011a,b), relies on intact functioning of several areas of the parietal cortex. Specifically, visual and verbal processing of quantity are posited to be based in the superior parietal lobule (SPL) and left angular gyrus, respectively, while the quantity system is localized bilaterally in the intraparietal sulcus (IPS; Menon et al., 2000; Naccache and Dehaene, 2001; Dehaene et al., 2003). This area is hypothesized to support number processing irrespective of the notation used.

The IPS is a prominent feature in the parietal lobe that runs from the postcentral sulcus posteriorly and into the occipital lobe, horizontally dividing the superior and inferior parietal lobule (IPL; Clark et al., 2010; Rubin and Safdieh, 2016). Along its route, the IPS is frequently interrupted and has a variable pattern of branching both on the medial and lateral (LIPS) walls (Cunningham, 1890; Zlatkina and Petrides, 2014).

Given the recognized role of the IPS in number sense, we were interested in whether the poorer arithmetic performance observed in children prenatally exposed to alcohol is related to alterations in the structure Sattlerregional. Since automated image segmentation tools to date have not captured patterns of morphological variation in the IPS, nor anatomically defined sub-regions of the IPS, we performed a manual tracing on magnetic resonance images to obtain volumes for the lateral and medial walls of the IPS. The present study examined: (1) the effects of PAE on IPS regional volumes and asymmetry, (2) whether IPS regional volumes are related to number processing performance and, if so, whether these relations are moderated by PAE, and (3) potential mediation by regional IPS volumes of the known relation between PAE and number processing performance.

\section{MATERIALS AND METHODS}

\section{Participants}

Participants were 52 right-handed, 9- to 14-year-old children from a historically disadvantaged community in Cape Town, South Africa, who are participating in our research on the effects of PAE on brain structure and function, cognition, and behavior (Jacobson et al., 2008; Meintjes et al., 2010; Jacobson et al., 2011c). All are from the same ethnic group. Within this sample, 15 had been diagnosed with FAS or PFAS, 13 were non-syndromal heavily exposed (HE) and 24 were non- or minimally exposed controls. The HE group consists of nonsyndromal children with confirmed heavy PAE who failed to meet the criteria for FAS or PFAS. Due to the small sample sizes, children with FAS or PFAS were grouped together. H.E. Hoyme (HEH), MD, and L.K. Robinson (LKR), MD, two U.S.-based FAS dysmorphologists examined each child at a clinic organized in September 2005 for growth and FAS dysmorphology using the Hoyme et al. (2005) protocol. N. Khaole, MD, a Cape Town-based dysmorphologist, examined the two children who were not able to attend the clinic. Considerable agreement between the examiners was reached on all the dysmorphic features assessed (Jacobson et al., 2008) and a consensus on FAS and PFAS diagnoses was reached during a case conference which included the dysmorphologists and SJ, JJ and $\mathrm{CM}$. The diagnoses have since been confirmed when the children were re-examined by HEH and LKR in 2009 and by a team of dysmorphologists led by $\mathrm{HEH}$ in follow-up clinics in 2013 and 2016 (see Jacobson et al., 2021).

Of the 52 participants, 20 were children in our prospective Cape Town Longitudinal Cohort study (Jacobson et al., 2008). The mothers of these children were recruited between July 1999 and January 2002 from an antenatal clinic of a midwife obstetric unit that serves an economically disadvantaged community. Mothers were invited to participate if they averaged at least $1.0 \mathrm{oz}$ absolute alcohol $(\mathrm{AA})$ per day $(\approx 2$ standard drinks), or reported at least two incidents of binge drinking (>5 standard drinks/occasion) during the first trimester of pregnancy. Additionally, a random sample of women from the same community initiating antenatal care around the same time was recruited as controls if they drank $<0.5 \mathrm{oz} \mathrm{AA}$ /day and if they did not binge drink during the first trimester. Mothers were excluded from the study if they were younger than 18 years of age, had a multiple gestation pregnancy, or suffered from chronic illnesses, such as diabetes, epilepsy, or cardiac problems, 
requiring treatment. Infants were excluded if they presented with major syndromes, neural tube defects, seizures, or if they were part of a multiple-gestation pregnancy or very low birth weight $(<1,500 \mathrm{~g})$ or GA $<32$ weeks. Prospective alcohol exposure data are available for the children from this cohort based on timeline follow-back interviews (Jacobson et al., 2002) conducted with their mothers during pregnancy. The additional 32 participants came from our cross-sectional cohort, who were identified by screening children from an elementary school in a nearby rural section of Cape Town where there is a high level of alcohol use among local farmworkers (Jacobson et al., 2011b). As with the longitudinal sample, two groups of children were recruited: (1) children whose mothers reported heavy drinking during pregnancy, i.e., who consumed at least 14 standard drinks/week (1.0 oz AA/day) on average or who engaged in binge drinking (5 or more drinks/occasion), and (2) controls whose mothers abstained or drank only minimally during pregnancy ( $\leq 2$ drinks per occasion).

\section{Procedure}

Mothers and children were transported in our research van to our Child Development Research Laboratory at the Faculty of Health Sciences, University of Cape Town (UCT), for neurobehavioral assessment and on the following day to the Cape Universities Brain Imaging Centre (CUBIC) at Tygerberg Hospital for scanning. Each mother completed a written informed consent, and each child provided assent. Breakfast, lunch, and snacks were provided for the mothers and children at each of their visits. The mother received monetary compensation for every visit, and each child received a small gift. Neuropsychological and neuroimaging assessments were administered to each child by examiners who were blind regarding maternal alcohol history and the child's FASD diagnosis, except for a few severe cases where the FAS diagnosis was apparent. Ethics approval for the study was obtained from the human research ethics committees at Wayne State University and the UCT Faculty of Health Sciences.

\section{Neuropsychological Assessment}

IQ was estimated using 7 of the 10 subtests from the Wechsler Intelligence Scale for Children, Third Edition (WISCIII)-Similarities, Arithmetic, Digit Span, Symbol Search, Coding, Block Design, and Picture Completion-and Matrix Reasoning from the WISC-IV. Sattler's (1992) formula for computing Short Form IQ was used to estimate the IQ of the children using the aforementioned subtests. Validity coefficients for Sattler Short Form IQ based on five or more subtests consistently exceed $r=0.90$ (Sattler, 1992). WISC Arithmetic and Digit Span scores were used as a measure of number processing performance. Handedness was assessed on the (Annett, 1970) Behavioral Handedness Inventory.

\section{Neuroimaging Assessment}

All children completed neuroimaging on a 3T Allegra MRI scanner (Siemens Medical Systems, Erlangen, Germany) using the single-channel head coil. High-resolution T1 weighted images of the participants from the longitudinal cohort were acquired in the sagittal plane using a volumetric navigated (Tisdall et al., 2012) multi-echo magnetization prepared rapid gradient echo (MEMPRAGE; Van Der Kouwe et al., 2008) sequence $\left(\right.$ FOV $256 \times 256 \times 167 \mathrm{~mm}^{3}, 128$ sagittal slices, TR $2,530 \mathrm{~ms}$, TE $1.53 / 3.21 / 4.89 / 6.57 \mathrm{~ms}$, TI $1,100 \mathrm{~ms}$, flip angle $7^{\circ}$, $\left.1.3 \times 1.0 \times 1.3 \mathrm{~mm}^{3}, 8.07 \mathrm{~min}\right)$. High-resolution anatomical images for the 32 participants of the cross-sectional cohort were also acquired in the sagittal plane using a magnetization prepared rapid gradient echo (MPRAGE) sequence (FOV $256 \times 256 \times 160 \mathrm{~mm}^{3}$, TR 2,300 ms, TE $3.93 \mathrm{~ms}$, TI $1,100 \mathrm{~ms}$, 160 slices, flip angle $\left.12^{\circ}, 1.3 \times 1.0 \times 1.0 \mathrm{~mm}^{3}, 6.03 \mathrm{~min}\right)$.

\section{MRI Analysis}

The IPS was manually traced on MR images displayed on a Wacom Cintiq tablet using a stylus and MultiTracer software (Woods, 2003). A protocol for manually tracing the cortical volume of the IPS on MR images was developed by CMRW, the senior neuroanatomist at UCT, and MM (Figure 1). All tracings were performed by a single investigator (MM) under the supervision of and in consultation with CW; no computer algorithms were employed to make or edit boundary decisions. A random subset of 10 brains was re-traced by the same investigator to examine intra-rater reliabilities.

The tracing protocol comprised two parts for each hemisphere: (1) tracing all the medial walls of the IPS (MIPS) and (2) tracing the entire sulcus by adding the lateral wall (LIPS). On sagittal and axial planes, we first identified the postcentral and parieto-occipital sulci to delimit the anterior and posterior boundaries of the parietal portion of the IPS (Figures 1A,B,E). The occipital portion was excluded. The IPS was initially traced on the axial sections where the patterns and branches were more clearly visible (Figure 1C). These tracings were then used to guide the tracing on the coronal sections.

MultiTracer software generates volumes for all manually delineated structures. We used "frust" volumes computed from the coronal tracings in each hemisphere of the MIPS and the entire IPS. "Frust" volumes are calculated by assuming that the structure extends from the center of the first plane on which it was drawn to the center of the last plane on which it was drawn with the square root of areas varying linearly when moving from the center of one plane to the center of the next (Woods, 2003). The volume of the LIPS was calculated by subtracting the volume of the MIPS from the total sulcal volume. A prominent medial branch was consistently present posteriorly for the MIPS and was included in the measurements (Figure 1D). Other sulci from the SPL that joined the MIPS in a few cases were excluded. All sulci branching from the LIPS into the inferior parietal lobule (IPL) were kexcluded.

\section{Statistical Analyses}

Statistical analyses were performed using SPSS (version 25, IBM). All variables were normally distributed, except for AA/day which was positively skewed and was log-transformed. The following variables had outliers greater than $3 \mathrm{SD}$ beyond the mean that were recoded to one unit greater than the next highest or lowest value (Winer, 1971): cigarettes/day $(n=1)$, degree of lead exposure $(n=1)$, AA/occasion $(n=1)$ and frequency of drinking days/week $(n=2)$. The alpha level for significance was set at 0.05 . 
A

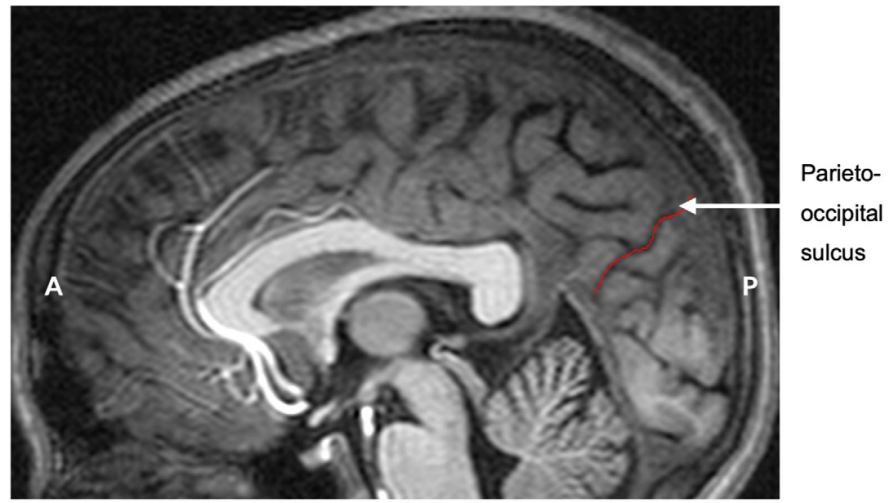

B

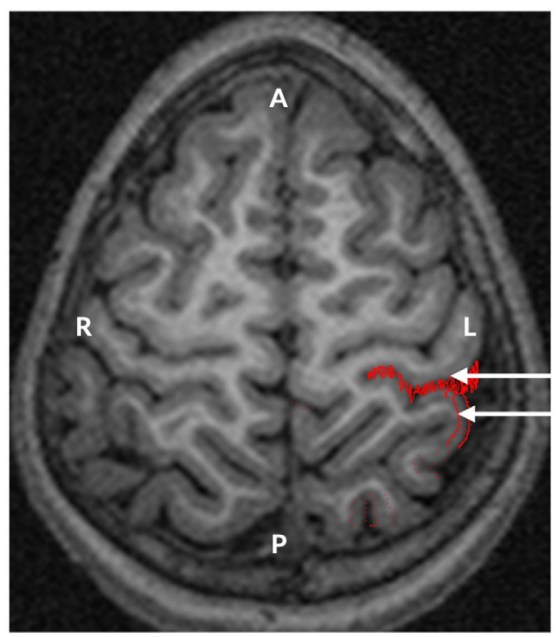

D

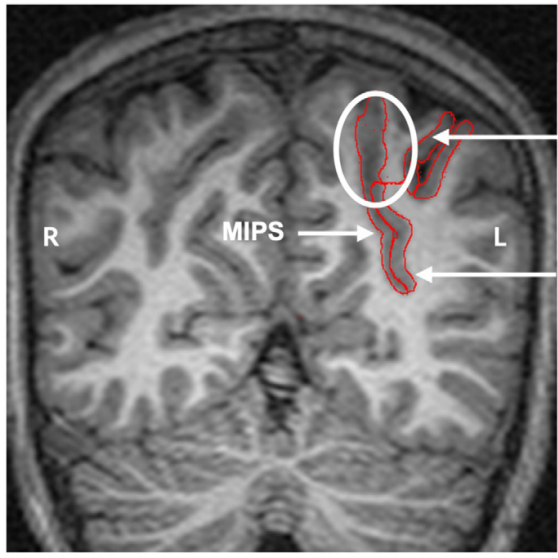

Postcentral

sulcus indicator

line

Outline

indicating the

start of the IPS

C

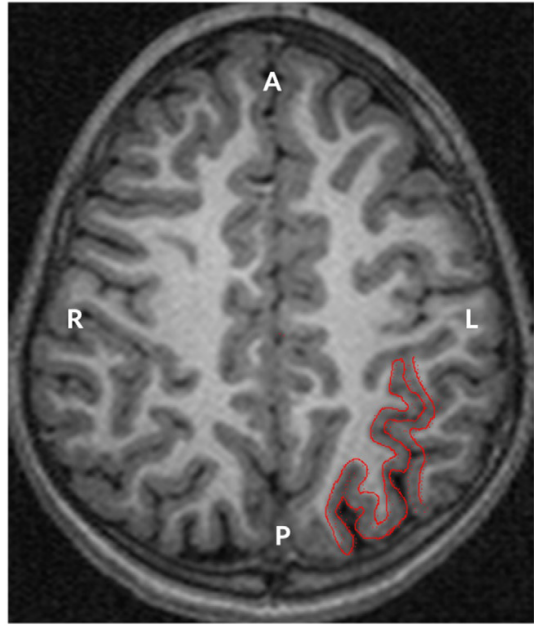

E

MIPS with

prominent

medial

branch

LIPS

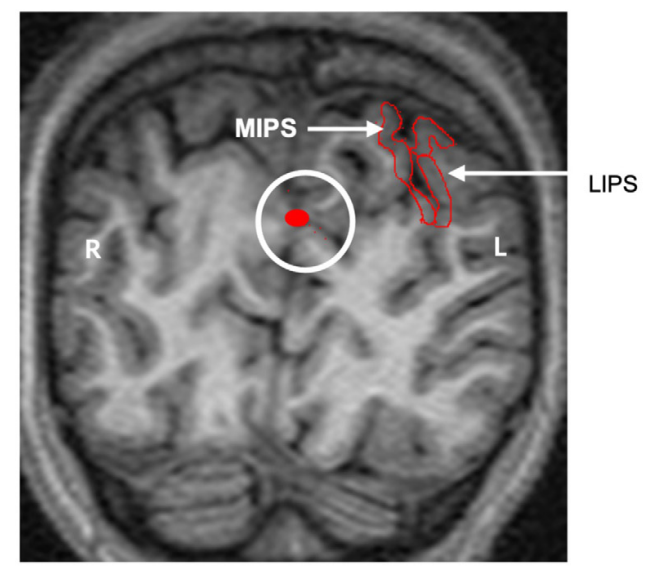

FIGURE 1 | The protocol for manually parcellating the intraparietal sulcus (IPS) is shown on the left hemisphere of a child with fetal alcohol syndrome (FAS)/partial FAS (PFAS). Both the medial (MIPS) and lateral (LIPS) walls of the IPS were traced. (A) The parieto-occipital sulcus was first identified on the sagittal plane to indicate the end of the parietal portion of the IPS. Tracings on the transverse planes serve to indicate (B) the start of the IPS at the postcentral sulcus and (C) the pattern of the sulcus. (D) The IPS was finally traced on the coronal sections. The white ellipse indicates the medial branch that was always present near the end of the parietal portion. The thin red line connecting the two sulci is required by the software for the total volume calculation as the area traced must be one continuous structure.

(E) The white circle indicates tracings from sagittal slices that are visible on the coronal planes, clearly indicating the parieto-occipital border of the IPS (A, Anterior; P, Posterior; L, Left hemisphere; R, Right hemisphere).

Intra-rater reliabilities were assessed using Pearson correlation and intraclass correlation (ICC) based on a mean-rating two-way mixed-effects model. We report ICC estimates for absolute agreement (Koo and Li, 2016).
Analysis of variance (ANOVA) was used to examine the differences in IPS regional volumes between the FASD diagnostic groups (FAS/PFAS, HE, controls), and between the most severely affected children with FAS or PFAS and controls. Six 
TABLE 1 | Sample characteristics.

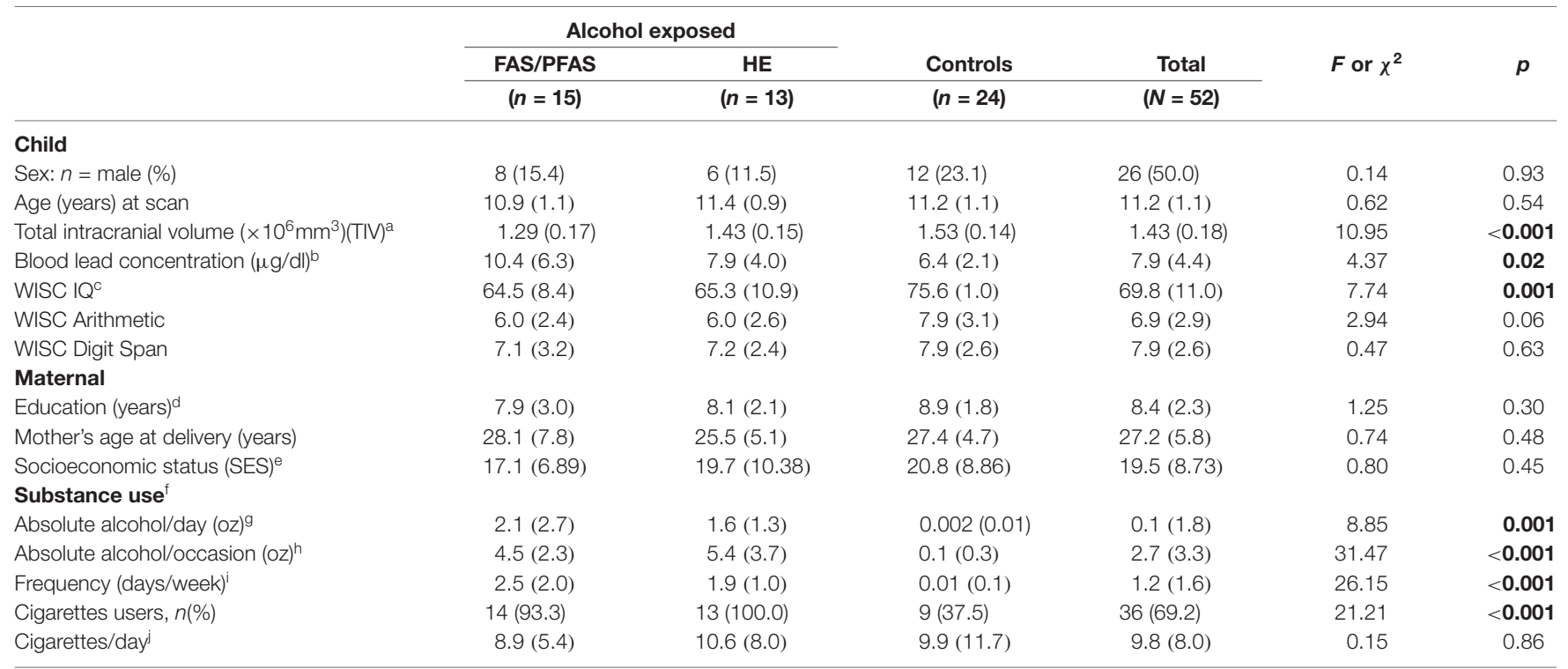

All variables are Mean (SD). FAS, fetal alcohol syndrome; PFAS, partial FAS; HE, nonsyndromal heavy alcohol exposed; WISC, Wechsler Intelligence Scale for Children; 1 oz absolute alcohol $\approx 2$ standard drinks; ${ }^{a}$ FAS/PFAS $<$ Controls $(p<0.001) ;{ }^{b}$ FAS/PFAS $>$ Controls $(p=0.013) ;{ }^{c}$ FAS $/ P F A S<$ Controls $(p=0.004) ; H E<$ Controls $(p=0.01) ;{ }^{d}$ Of $N=52,48$ primary caregivers were the biological mothers of the children; 2 (HE) were the fathers, 1 (Control) was the grandmother, and 1 (FAS/PFAS) was another relative; ${ }^{e}$ Hollingshead (2011) Four Factor Index of Socioeconomic Scale; ${ }^{f}$ One mother of a child with FAS used marijuana 0.87 days/month during pregnancy; ${ }^{g} F A S / P F A S>C o n t r o l s ~(p=0.001)$, HE > Controls $(p=0.016) ;{ }^{h}$ FAS/PFAS, HE > Controls $(p<0.001) ;{ }^{i}$ FAS/PFAS, HE > Controls $(p<0.001) ;{ }^{j}$ Smokers only. Bold denotes significance at $p<0.05$.

control variables were considered and assessed as potential confounders: MRI sequence used, child sex and age at scan (years), socioeconomic status (Hollingshead, 2011), maternal cigarette smoking during pregnancy (number of cigarettes smoked/day) and child lead exposure ( $\mu \mathrm{g} / \mathrm{dl})$. Using Pearson $r$, we examined the association of each of these control variables with the outcome variables. Any control variable showing even a weak association (at $p<0.10$ ) with an outcome variable was controlled for in subsequent analyses with that outcome variable using ANCOVA or multiple regression, respectively. We also present results both before and after adjustment for total intracranial volume (TIV) to examine whether effects of PAE on IPS regional volumes demonstrate a specific vulnerability to the teratogenic effects of PAE or to what degree they are attributable to PAE-related reductions in overall brain size. Additionally, potential effects of PAE on brain asymmetry were examined by comparing regional volumes in left and right hemispheres separately within each diagnostic group.

The relationship between IPS regional volumes and number processing performance was first assessed using Pearson $r$. Subsequently, we examined whether PAE moderates the relation between IPS regional volumes and number processing performance by conducting hierarchical multiple regression analyses. In the first step, the IPS regional volume and PAE were included, and in the second step, the interaction term between the IPS volume and PAE was added to the regression model.

Finally, we performed a series of hierarchical multiple regressions to examine whether IPS regional volumes mediate the effect of PAE on number processing. AA/day during pregnancy was entered in the first step of each regression; the IPS regional volume was entered in the second step. Mediation was inferred if the addition of the IPS volume substantially reduced the magnitude of the regression coefficient for AA/day (Baron and Kenny, 1986). The difference in coefficient tests (Sobel, 1982) was used to assess whether the reduction was statistically significant.

\section{RESULTS}

Sample characteristics are shown in Table 1. There were no between-group differences in terms of child sex and age, maternal education, age, or socioeconomic status. Children in the FAS/PFAS group had smaller TIV and higher blood lead concentrations than controls, and children in both alcoholexposed groups had lower WISC IQ scores than controls. Although below conventional levels of significance, children in the FAS/PFAS and HE groups performed more poorly on WISC Arithmetic subtest than controls but not on WISC Digit Span. All but two mothers of the control children abstained from drinking: one consumed two drinks on one occasion; the other, two drinks about once/month. Although more of the mothers in the FAS/PFAS and HE groups smoked cigarettes than controls, there were no significant group differences among smokers regarding the number of cigarettes smoked/day, all $p$ 's $>0.25$. One mother of a child in the FAS/PFAS group reported smoking marijuana about 1 day/month across pregnancy. None of the other mothers reported using marijuana, and none reported using cocaine, methaqualone, or any other illicit drugs.

Intra-rater reliabilities are presented in Table 2. As per the convention proposed by Koo and Li (2016), volumes of all regions demonstrated good reliabilities (ICC estimates between 0.75 and 0.9 ), except for the right LIPS where it was moderate (ICC estimate between 0.5 and 0.75 ).

Table 3 shows the associations of volumes in each region with TIV and with potential confounders. Imaging sequence, 
TABLE 2 | Intra-rater reliability of the ROI volumes for 10 randomly selected participants.

\begin{tabular}{llcl}
\hline & \multicolumn{1}{c}{ Pearson } & ICC (absolute agreement) & \multirow{2}{*}{$\mathbf{9 5 \%} \mathbf{~ C l}$} \\
\cline { 2 - 3 } & \multicolumn{1}{c}{$\boldsymbol{r}(\boldsymbol{p})$} & $\boldsymbol{r}(\boldsymbol{p})$ & \\
\hline Left MIPS & $0.774(\mathbf{0 . 0 0 9 )}$ & $0.757(\mathbf{0 . 0 0 4 )}$ & $0.286-0.934$ \\
Right MIPS & $0.906(\mathbf{0 . 0 0 1 )}$ & $0.850(\mathbf{0 . 0 0 1 )}$ & $0.449-0.962$ \\
Left LIPS & $0.809(\mathbf{0 . 0 0 5 )}$ & $0.821(\mathbf{0 . 0 0 1 )}$ & $0.426-0.953$ \\
Right LIPS & $0.696(\mathbf{0 . 0 2 5 )}$ & $0.694(\mathbf{0 . 0 1 )}$ & $0.163-0.914$
\end{tabular}

MIPS, Medial wall; LIPS, Lateral wall, ICC, Intraclass correlation coefficient. Bold denotes significance at $p<0.05$.

child sex, and smoking each showed an association with one ROI volume. By contrast, TIV was related to three of the four ROI volumes. Although lead exposure levels in children in the FAS/PFAS group were higher than in either of the other two groups, it showed no association with any of the volumes examined here and was, therefore, not controlled for in subsequent analyses.

As shown in Table 4 and Figure 2, there was a significant association between the FASD group and left LIPS volumes, even after control for potential confounders. Adjustment for TIV reduced the association between group and left LIPS volume to below conventional levels of significance, suggesting that the effect reflected, in part, smaller overall brain volume in the FAS/PFAS group compared to controls. Direct comparison of the most severely affected children in the FAS/PFAS group and controls revealed that the left LIPS volumes were significantly smaller in children with FAS/PFAS than in controls $(t=3.17, p=0.003)$, even after adjustment for potential confounders $(F=6.16, p=0.02)$, $\operatorname{TIV}(F=5.52, p=0.02)$, or both $(F=4.48, p=0.04)$. Although the association between FASD diagnosis and left MIPS volumes just missed conventional levels of significance $(p=0.06)$ and was no longer even a trend after inclusion of the potential confounders or adjustment for TIV (Table 4), direct comparison of the most severely affected children in the FAS/PFAS and control groups indicated that left MIPS was significantly smaller for the FAS/PFAS group than for controls $(t=2.32, p=0.03)$ but that this finding does not survive after control for smoking $(F=2.54, p=0.12)$ or $\operatorname{TIV}(F=1.35, p=0.25)$. Volumes of the right MIPS and right LIPS did not differ between children in the FAS/PFAS and control groups $(p=0.22$ and $p=0.09$, respectively).

While no left-right asymmetry was evident in the medial wall of the IPS in any of the groups, asymmetry in the lateral IPS was seen in control children that were not evident in children with FAS/PFAS, and evident below conventional levels of significance in the HE group (Table 5).

Larger left and right MIPS were associated with higher WISC Arithmetic scores (Table 6, Figures 3A,B). While multiple regression showed that both MIPS volumes and PAE affect WISC Arithmetic performance, PAE did not moderate the relations between MIPS volumes and WISC Arithmetic. Regional IPS volumes also did not mediate the effect of PAE on WISC Arithmetic (Figure 4).

\section{DISCUSSION}

In this study we manually traced the IPS on MR images to investigate the effects of PAE on regional IPS volumes and whether alterations in IPS volume play a role in number processing deficits observed in PAE (Meintjes et al., 2010; Woods et al., 2015). While both the left lateral and medial walls were smaller in children in the FAS/PFAS group compared to controls, group differences in the medial wall did not survive after control for smoking or TIV. Notably, the asymmetric larger volumes of the left LIPS compared to the right seen in control children were not evident in children with FAS/PFAS, which may account for volume reductions seen in these children in this region. Although bilaterally larger medial walls were associated with better performance on the WISC Arithmetic test-effects that were not moderated by PAE-regional IPS volumes did not mediate the effect of PAE on WISC Arithmetic.

TABLE 3 | Associations of volumes with TIV and potential confounders $(N=52)$.

\begin{tabular}{|c|c|c|c|c|c|c|c|c|c|c|c|c|c|c|}
\hline & \multicolumn{2}{|c|}{$\begin{array}{c}\text { Total intracranial } \\
\text { volume }\end{array}$} & \multicolumn{2}{|c|}{ Imaging $^{1}$} & \multicolumn{2}{|c|}{ Age at scan } & \multicolumn{2}{|c|}{ Sex of child ${ }^{1}$} & \multicolumn{2}{|c|}{$\begin{array}{c}\text { Socio-economic } \\
\text { status }\end{array}$} & \multicolumn{2}{|c|}{ Cigarettes/day ${ }^{2}$} & \multicolumn{2}{|c|}{ Lead exposure $^{2}$} \\
\hline & $r$ & $p$ & $r$ & $p$ & $r$ & $p$ & $r$ & $p$ & $r$ & $p$ & $r$ & $p$ & $r$ & $p$ \\
\hline Left MIPS & 0.38 & 0.005 & 0.11 & 0.46 & -0.18 & 0.20 & 0.01 & 0.92 & 0.04 & 0.77 & -0.24 & 0.08 & -0.17 & 0.23 \\
\hline Right MIPS & 0.27 & 0.05 & 0.08 & 0.56 & -0.16 & 0.25 & -0.00 & 0.98 & -0.11 & 0.44 & 0.02 & 0.88 & -0.05 & 0.73 \\
\hline Left LIPS & 0.26 & 0.06 & 0.31 & 0.03 & -0.05 & 0.72 & 0.25 & 0.07 & -0.07 & 0.61 & -0.18 & 0.20 & -0.22 & 0.11 \\
\hline Right LIPS & 0.22 & 0.11 & 0.22 & 0.11 & -0.15 & 0.30 & 0.08 & 0.58 & -0.05 & 0.74 & 0.04 & 0.80 & -0.05 & 0.73 \\
\hline
\end{tabular}

Values are Pearson correlation coefficients. ${ }^{1}$ For categorical variables, point biserial correlation was used. ${ }^{2}$ One outlier (>3 SD beyond the mean) recoded to 1 unit higher than the next highest value. Bold denotes association at $p<0.10$. MIPS, Medial wall; LIPS, Lateral wall; TIV, total intracranial volume.

TABLE 4 | Comparison of volumes by FASD diagnosis $(N=52)$.

\begin{tabular}{|c|c|c|c|c|c|c|c|c|c|}
\hline & FAS/PFAS $(n=15)$ & HE $(n=13)$ & Controls $(n=24)$ & & & & & & \\
\hline & Mean (SD) & Mean (SD) & Mean (SD) & $\boldsymbol{F}$ & $p$ & $F^{1}$ & $p^{1}$ & $F^{2}$ & $p^{2}$ \\
\hline Left MIPSa & $5,458(1,169)$ & $6,128(995)$ & $6,528(1,530)$ & 3.05 & 0.06 & 2.21 & 0.12 & 0.60 & 0.55 \\
\hline Right MIPS & $5,800(941)$ & $5,645(2,016)$ & $6,303(1,369)$ & 1.05 & 0.36 & 1.05 & 0.36 & 0.47 & 0.63 \\
\hline Left LIPS ${ }^{b, c}$ & $2,871(670)$ & $3,648(1,023)$ & $3,758(941)$ & 4.85 & $0.01^{*}$ & 3.95 & 0.03 & 2.90 & 0.07 \\
\hline Right LIPS & $2,668(726)$ & $2,915(1,038)$ & $3,211(1,072)$ & 1.46 & 0.24 & 1.46 & 0.24 & 0.47 & 0.62 \\
\hline
\end{tabular}

${ }^{1}$ ANCOVA controlling for potential confounders; FAS, fetal alcohol syndrome; PFAS, partial FAS; HE, nonsyndromal heavy alcohol exposed; FASD, fetal alcohol spectrum disorders. ${ }^{2}$ ANCOVA controlling for TIV. Potential confounders are a Cigarettes/day, ${ }^{b}$ Imaging sequence, ${ }^{c}$ Sex of the child. * Tukey post hocs: Left LIPS: FAS/PFAS < Controls ( $\left.p=0.01\right)$. MIPS, Medial wall; LIPS, Lateral wall. Bold denotes significance at $p<0.05$. 
A

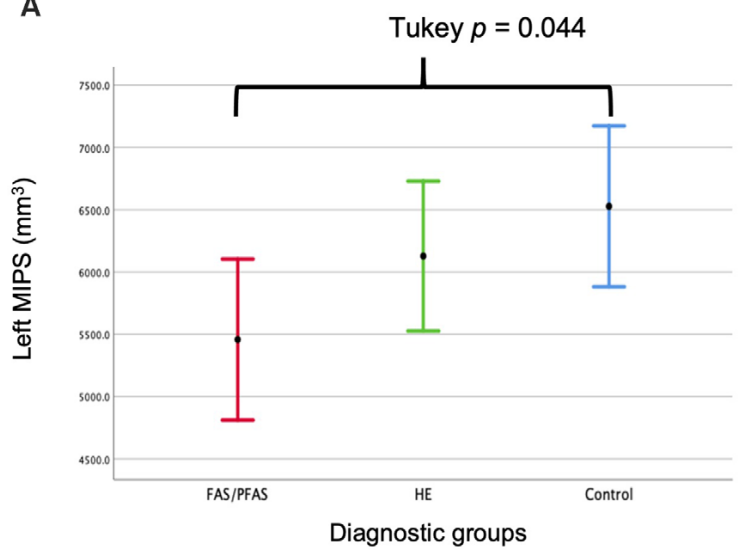

B

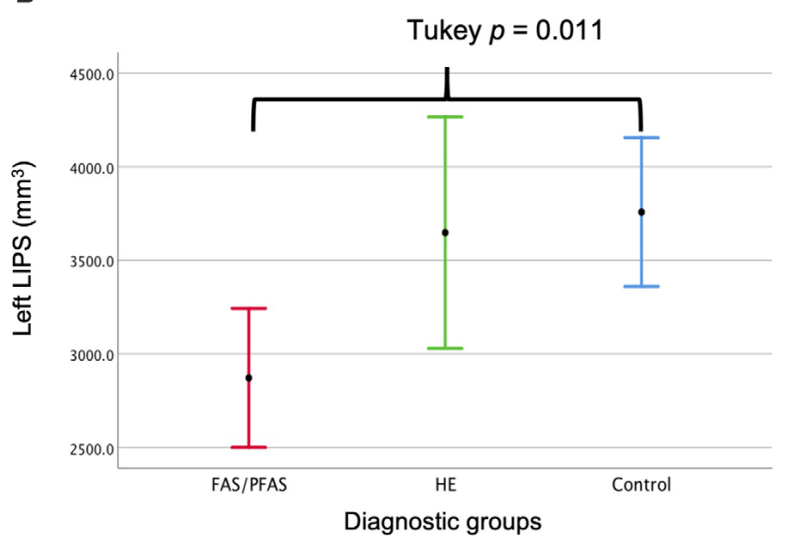

FIGURE 2 | Comparison of the (A) left medial (M) and (B) left lateral (L) IPS volumes by diagnostic group (mean \pm 95\% Confidence Intervals).

TABLE 5 | Comparison of hemispheric asymmetry by FASD diagnosis $(N=52)$.

\begin{tabular}{|c|c|c|c|c|c|c|c|c|c|}
\hline & \multicolumn{3}{|c|}{ FAS/PFAS $(n=15)$} & \multicolumn{3}{|c|}{ HE $(n=13)$} & \multicolumn{3}{|c|}{ Controls ( $n=24$ ) } \\
\hline & Mean (SD) & $t$ & $p$ & Mean (SD) & $t$ & $p$ & Mean (SD) & $t$ & $p$ \\
\hline Left MIPS & $5,458(1,168)$ & \multirow{2}{*}{-1.45} & \multirow{2}{*}{0.17} & 6,128 (995) & \multirow{2}{*}{1.22} & \multirow{2}{*}{0.25} & $6,528(1,530)$ & \multirow{2}{*}{0.95} & \multirow{2}{*}{0.35} \\
\hline Right MIPS & $5,800(941)$ & & & $5,645(2,016)$ & & & $6,303(1,369)$ & & \\
\hline Left LIPS & $2,871(670)$ & \multirow{2}{*}{1.12} & \multirow{2}{*}{0.28} & $3,648(1,023)$ & \multirow{2}{*}{2.10} & \multirow{2}{*}{0.06} & 3,758 (941) & \multirow{2}{*}{2.53} & \multirow{2}{*}{0.02} \\
\hline Right LIPS & 2,668 (726) & & & $2,915(1,038)$ & & & $3,211(1,072)$ & & \\
\hline
\end{tabular}

FAS, fetal alcohol syndrome; PFAS, partial FAS; HE, nonsyndromal heavy alcohol exposed. MIPS, Medial wall; LIPS, Lateral wall. Bold denotes significance at p < 0.05.

TABLE 6 | Relation between math performance on the WISC Arithmetic and regional IPS volumes, before and after adjustment for degree of prenatal alcohol exposure and potential moderation by prenatal alcohol exposure of the relations between regional IPS volumes and WISC Arithmetic.

\begin{tabular}{|c|c|c|c|c|c|c|}
\hline & \multirow[b]{2}{*}{$r(p)$} & \multicolumn{2}{|c|}{ Model 1} & \multicolumn{3}{|c|}{ Model 2} \\
\hline & & $\beta^{1}(p)$ & $\beta^{2}(p)$ & $\beta^{1 \prime}(p)$ & $\beta^{2 \prime}(p)$ & $\beta^{3}(p)$ \\
\hline Left MIPS & $0.35(0.01)$ & $0.29(0.02)$ & $-0.39(0.002)$ & $0.28(0.03)$ & $-0.40(0.002)$ & $-0.09(0.46)$ \\
\hline Right MIPS & $0.30(0.03)$ & $0.27(0.03)$ & $-0.41(0.002)$ & $0.27(0.03)$ & $-0.42(0.001)$ & $-0.17(0.18)$ \\
\hline Left LIPS & $0.04(0.81)$ & $-0.02(0.85)$ & $-0.44(0.002)$ & $-0.03(0.85)$ & $-0.44(0.002)$ & $0.004(0.98)$ \\
\hline Right LIPS & $0.02(0.87)$ & $-0.004(0.98)$ & $-0.43(0.002)$ & $0.004(0.98)$ & $-0.43(0.002)$ & $0.02(0.88)$ \\
\hline
\end{tabular}

r, Pearson correlation coefficient; Model 1: WISC Arithmetic $=\beta^{0}+\beta^{1}($ ROI Volume $)+\beta 2(A A$ day $) ;$ Model $2:$ WISC Arithmetic $=\beta^{0}+\beta^{1 \prime}(R O I$ Volume $)+\beta^{21}(A A / d a y)+\beta^{3}(R O I$ volume*AA/day). MIPS, Medial wall; LIPS, Lateral wall; WISC, Wechsler Intelligence Scale for Children. Bold denotes significance at $p<0.05$.

Although numerous studies have examined the effects of PAE on brain volumes (see Lebel et al., 2011 review), none have focused specifically on the IPS. Our results extend findings from Archibald et al. (2001) of parietal lobe reductions in 11- to 13-year-old children with FAS to a specific structure. Similar to Sowell et al. (2001), who found structural abnormalities in PAE individuals aged 8-22 years predominantly in the left posterior temporo-parietal cortices, we also found only the left IPS to be smaller in children in the FAS/PFAS group. Although both the medial and lateral walls were smaller, reductions in the medial wall did not survive after controlling for maternal smoking during pregnancy. Prenatal exposure to smoking has been associated with reduced brain volumes of the frontal lobe and cerebellum (Ekblad et al., 2010), as well as increased activity in the left SPL during tasks involving working memory (Longo et al., 2014). Alternatively, compared with alcohol recall, smoking is more habitual and, therefore, easier to recall (Jacobson et al.,
2002). It is also more readily quantifiable than alcohol and may, therefore, account for more of the shared variance between these exposures and the outcome. Thus, analyses controlling for alcohol may overattribute deficits to smoking and under control for the effect of alcohol exposure.

Lesion and imaging studies have confirmed the role of the IPS in arithmetic and number processing (Cohen and Dehaene, 1995; Menon et al., 2000; Isaacs et al., 2001; Naccache and Dehaene, 2001; Dehaene et al., 2003). Localizing the exact areas within the IPS involved in performing arithmetic tasks could aid in better understanding the impact of the PAE-related structural alterations on mathematical abilities and potentially developing remedial or pharmacological treatments. In our study, both the left- and right MIPS volumes but not the lateral walls were associated with WISC Arithmetic scores. Associations remained essentially unchanged after adjustment for the degree of alcohol exposure and potential interaction 

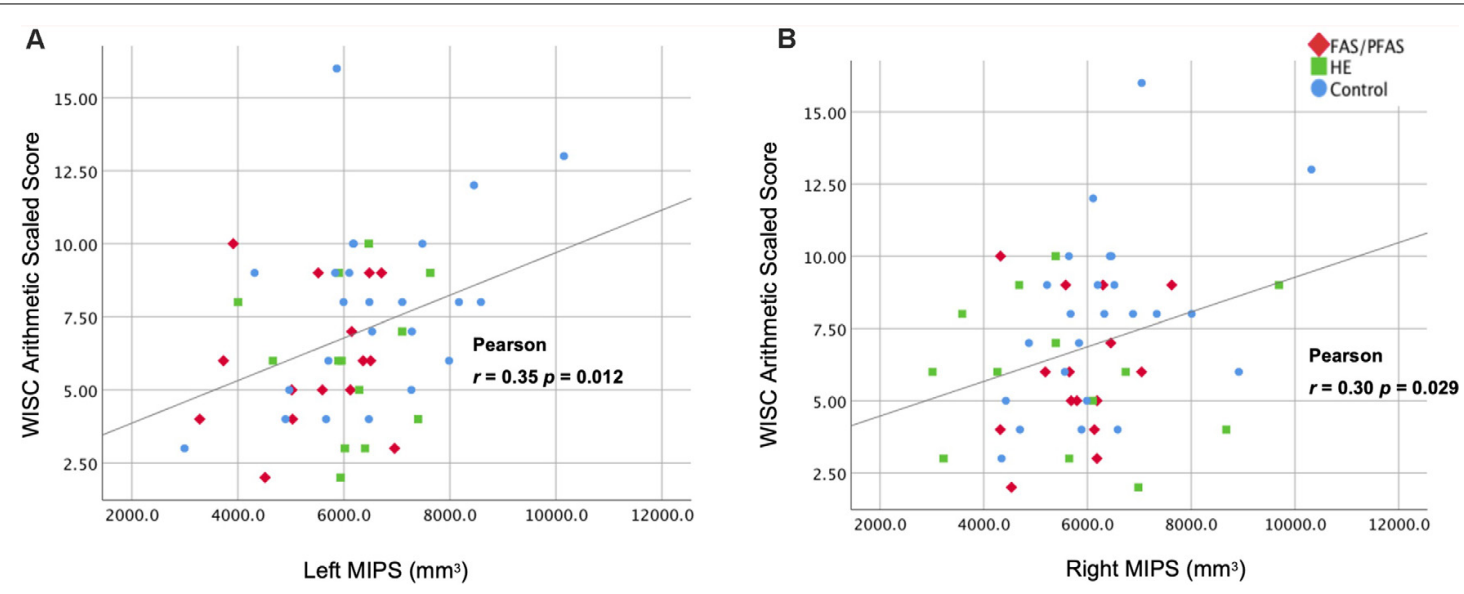

FIGURE 3 | Associations of WISC-IV Arithmetic scores with (A) left and (B) right medial IPS (MIPS) volumes.

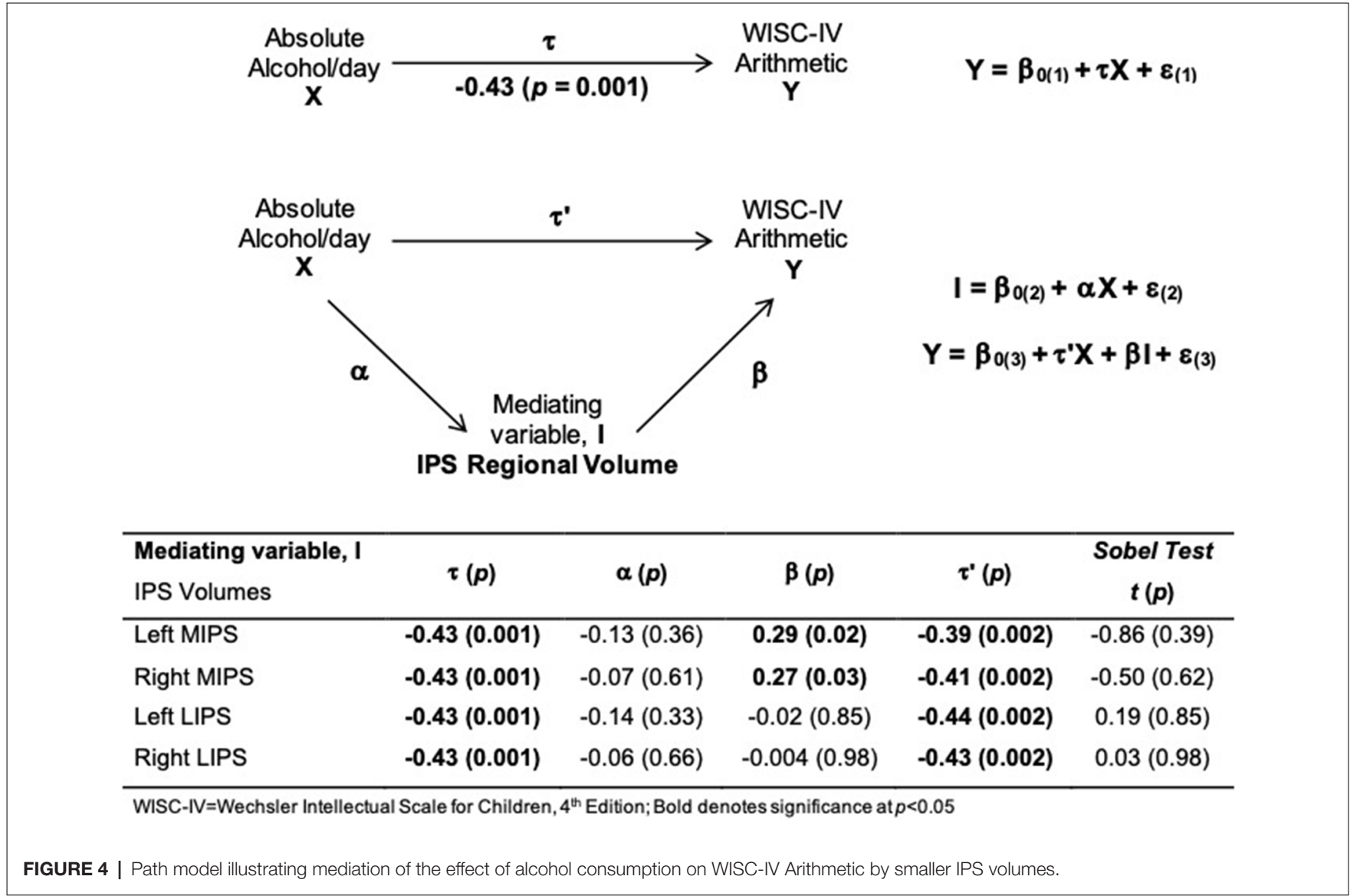

effects. Five key parietal areas for number processing have been suggested, including the left and right posterior SPL and the IPS, with its anterior portion being most active during the interpretation of magnitude (Dehaene et al., 2003). The location of the posterior SPL delineated by Dehaene et al. (2003) and used by Woods et al. (2015) in fMRI studies of number processing corresponds neuroanatomically with the medial branch of the
MIPS in the current study. The posterior SPL is activated during certain arithmetic calculations, such as counting (Piazza et al., 2003) and number comparison (Pinel et al., 2001). Since the posterior SPL and anterior portion of the IPS comprise most of our MIPS volumes, it is not surprising that children with larger MIPS volumes performed better on the WISC arithmetic assessments. 
During the process of manual tracing, extreme inter-subject variations were observed in the branching patterns and shapes of the sulcus between hemispheres. Interhemispheric asymmetry, in terms of volume, observed in the LIPS in controls and HE children were not seen in children in the FAS/PFAS group or in the MIPS in any of the FASD diagnostic groups. Structural asymmetry is known to be typical in healthy brains (Watkins et al., 2001). Although PAE-related changes in typical asymmetry have been reported previously (Sowell et al., 2002), this is the first study to assess the asymmetry of the IPS.

A limitation in this study was the small sizes of the FAS/PFAS and HE groups. Thus, the results would need to be confirmed in a larger independent study. Two MRI sequences (MPRAGE for $n=32$; and MEMPRAGE for $n=20$ ) were used for scanning, which may have altered the MR images slightly and could have affected the volume results. This was, however, adjusted for by including imaging sequence as a potential confounder. Since the children in our cohort were recruited from an educationally and socio-economically deprived community, our findings need to be examined in children raised in less deprived environments to see if these findings also hold for them. Strengths of the current study include the use of the timeline follow-back approach, which allowed the researchers to examine dose-dependent effects, and manual tracing, which allowed an expert neuroanatomist to make informed decisions related to branching and structural variations observed in the IPS.

This study used manual tracing on MR images to examine the effects of PAE on IPS volumes and associations with number processing. In contrast to other studies that examined volumes of the parietal lobe as a whole, our study examined the volumes of sub-regions within the IPS specifically. The left IPS was found to be smaller in children with FAS or PFAS, which fell below conventional levels of significance, after adjustment for overall reductions in brain size. This volume reduction may account for the loss of asymmetry seen in children with FAS and PFAS in the lateral wall. Bilaterally, increasing MIPS volumes were associated with better arithmetic performance on the WISC IQ test.

\section{DATA AVAILABILITY STATEMENT}

The raw data supporting the conclusions of this article will be made available by the authors, without undue reservation.

\section{REFERENCES}

Annett, M. (1970). A classification of hand preference by association analysis. $\mathrm{Br}$. J. Psychol. 61, 303-321. doi: 10.1111/j.2044-8295.1970.tb01248.x

Archibald, S. L., Fennema-Notestine, C., Gamst, A., Riley, E. P., Mattson, S. N., and Jernigan, T. L. (2001). Brain dysmorphology in individuals with severe prenatal alcohol exposure. Dev. Med. Child Neurol. 43, 148-154. doi: 10.1111/j.20448295.1970.tb01248.x

Baron, R. M., and Kenny, D. A. (1986). The moderator-mediator variable distinction in social psychological research: Conceptual, strategic and statistical considerations. Pers. Soc. Psychol. Rev. 51, 1173-1182. doi: 10.1037/0022-3514. 51.6.1173

Bertrand, J., Floyd, R. L., and Weber, M. K. (2005). Guidelines for identifying and referring persons with fetal alcohol syndrome. MMWR. Recomm. Rep. 54, 1-4.

\section{ETHICS STATEMENT}

The studies involving human participants were reviewed and approved by the University of Cape Town Faculty of Health Sciences Human Research Ethics Committee and the Wayne State University Institutional Review Board. Written informed consent to participate in this study was provided by the participants' legal guardian/next of kin.

\section{AUTHOR CONTRIBUTIONS}

MM together with CW designed the protocol for tracing the areas from which the volumes were calculated, and MM completed the tracings for all the subjects. She also conducted the review of the literature, statistical analyses, interpretation, and write-up of the findings. EM, FW, and CW provided supervision for MM's project and collaborated on the write-up, study design, and statistical analyses. SJ, JJ, and EM designed, implemented, oversaw the data collection on the Cape Town FASD study, collaborated on the statistical analyses, write-up, and interpretation of the findings from this study. Maternal interviews were administered by CM.

\section{FUNDING}

This study was supported by grants from the NIH/National Institute on Alcohol Abuse and Alcoholism (R01 AA09524, U01 AA014790, R01 AA016781, and R01 AA023503), South African National Research Foundation (NRF) Grant number: 48337, and the Lycaki-Young Fund, State of Michigan.

\section{ACKNOWLEDGMENTS}

We thank H. E. Hoyme, L. K. Robinson, and N. Khaole, who conducted the dysmorphology assessments; M. Pienaar, M. September, R. Sun, and N. Dodge, our University of Cape Town and Wayne State University research staff; and G. Adams, S. Heyne, P. Samuels, K. Salie, and N. Solomon, the MR technologists at the Cape Universities Brain Imaging Centre. We again thank the mothers and children for their long-term participation in our Cape Town Longitudinal Cohort study.

Burden, M. J., Jacobson, S. W., Sokol, R. J., and Jacobson, J. L. (2005). Effects of prenatal alcohol exposure on attention and working memory at 7.5 years of age. Alcohol Clin. Exp. Res. 29, 443-452. doi: 10.1097/01.alc.0000156125. 50577.ec

Clark, D. L., Boutros, N. N., and Mendez, M. F. (2010). The Brain and Behavior: an Introduction to Behavioral Neuroanatomy. New York, NY: Cambridge University Press.

Cohen, L., and Dehaene, S. (1995). Number processing in pure alexia: The effect of hemispheric asymmetries and task demands. Neurocase 1, 121-137. doi: $10.1093 /$ neucas/1.2.121-a

Croxford, J., and Viljoen, D. (1999). Alcohol consumption by pregnant women in the Western Cape. S. Afr. Med. J. 89, 962-965.

Cunningham, D. J. (1890). The intraparietal sulcus of the brain. J. Anat. Physiol. 24, 135-155. 
Dehaene, S., Piazza, M., Pinel, P., and Cohen, L. (2003). Three parietal circuits for number processing. Cogn. Neuropsychol. 20, 487-506. doi: 10.1080/02643290244000239

Ekblad, M., Korkeila, J., Parkkola, R., Lapinleimu, H., Haataja, L., Lehtonen, L., et al. (2010). Maternal smoking during pregnancy and regional brain volumes in preterm infants. J. Pediatr. 156, e181.185-e181.190. doi: 10.1016/j.jpeds. 2009.07.061

Hollingshead, A. B. (2011). Four factor index of social status. Yale J. Sociol. 8, 21-51.

Howell, K. K., Lynch, M. E., Platzman, K. A., Smith, G. H., and Coles, C. D. (2006). Prenatal alcohol exposure and ability, academic achievement and school functioning in adolescence: a longitudinal follow-up. J. Pediatr. Psychol. 31, 116-126. doi: 10.1093/jpepsy/jsj029

Hoyme, H. E., Kalberg, W. O., Elliott, A. J., Blankenship, J., Buckley, D., Marais, A. -S., et al. (2016). Updated clinical guidelines for diagnosing fetal alcohol spectrum disorders. Pediatrics 138:e20154256. doi: 10.1542/peds.20154256

Hoyme, H. E., May, P. A., Kalberg, W. O., Kodituwakku, P., Gossage, J. P., Trujillo, P. M., et al. (2005). A practical clinical approach to diagnosis of fetal alcohol spectrum disorders: clarification of the 1996 institute of medicine criteria. Pediatrics 115, 39-47. doi: 10.1542/peds.2004-0259

Infante, M. A., Moore, E. M., Bischoff-Grethe, A., Tapert, S. F., Mattson, S. N., and Riley, E. P. (2017). Altered functional connectivity during spatial working memory in children with heavy prenatal alcohol exposure. Alcohol 64, 11-21. doi: 10.1016/j.alcohol.2017.05.002

Isaacs, E., Edmonds, C., Lucas, A., and Gadian, D. (2001). Calculation difficulties in children of very low birthweight: a neural correlate. Brain 124, 1701-1707. doi: 10.1093/brain/124.9.1701

Jacobson, J. L., Dodge, N. C., Burden, M. J., Klorman, R., and Jacobson, S. W. (2011a). Number processing in adolescents with prenatal alcohol exposure and ADHD: differences in the neurobehavioral phenotype. Alcohol Clin. Exp. Res. 35, 431-442. doi: 10.1111/j.1530-0277.2010.01360.x

Jacobson, S. W., Carr, L. G., Croxford, J., Sokol, R. J., Li, T. -K., and Jacobson, J. L. (2006). Protective effects of the alcohol dehydrogenase-ADH1B allele in children exposed to alcohol during pregnancy. J. Pediatr. 148, 30-37. doi: 10.1016/j.jpeds.2005.08.023

Jacobson, S. W., Chiodo, L. M., Sokol, R. J., and Jacobson, J. L. (2002). Validity of maternal report of prenatal alcohol, cocaine and smoking in relation to neurobehavioral outcome. Pediatrics 109, 815-825. doi: 10.1542/peds. 109.5.815

Jacobson, S. W., Hoyme, H. E., Carter, R. C., Dodge, N. C., Molteno, C. D., Meintjes, E. M., et al. (2021). Evolution of the physical phenotype of fetal alcohol spectrum disorders from childhood through adolescence. Alcohol Clin. Exp. Res. 45, 395-408. doi: 10.1111/acer.14534

Jacobson, S. W., Jacobson, J. L., Stanton, M. E., Meintjes, E. M., and Molteno, C. D. (2011b). Biobehavioral markers of adverse effect in fetal alcohol spectrum disorders. Neuropsychol. Rev. 21, 148-166. doi: 10.1007/s11065-011-9169-7

Jacobson, S. W., Stanton, M. E., Dodge, N. C., Pienaar, M., Fuller, D. S., Molteno, C. D., et al. (2011c). Impaired delay and trace eyeblink conditioning in school-age children with fetal alcohol syndrome. Alcohol Clin. Exp. Res. 35, 250-264. doi: 10.1111/j.1530-0277.2010.01341.x

Jacobson, S. W., Stanton, M. E., Molteno, C. D., Burden, M. J., Fuller, D. S., Hoyme, H. E., et al. (2008). Impaired eyeblink conditioning in children with fetal alcohol syndrome. Alcohol Clin. Exp. Res. 32, 365-372. doi: 10.1111/j.15300277.2007.00585.x

Jones, K. L., and Smith, D. W. (1973). Recognition of the fetal alcohol syndrome in early infancy. Lancet 302, 999-1001. doi: 10.1016/s0140-6736(73)91092-1

Koo, T. K., and Li, M. Y. (2016). A guideline of selecting and reporting intraclass correlation coefficients for reliability research. J. Chiropr. Med. 15, 155-163. doi: 10.1016/j.jcm.2016.02.012

Kopera-Frye, K., Dehaene, S., and Streissguth, A. P. (1996). Impairments of number processing induced by prenatal alcohol exposure. Neuropsychologia 34, 1187-1196. doi: 10.1016/0028-3932(96)00043-7

Lebel, C., Mattson, S. N., Riley, E. P., Jones, K. L., Adnams, C. M., May, P. A., et al. (2012). A longitudinal study of the long-term consequences of drinking during pregnancy: heavy in utero alcohol exposure disrupts the normal processes of brain development. J. Neurosci. 32, 15243-15251. doi: 10.1523/JNEUROSCI. 1161-12.2012
Lebel, C., Roussotte, F., and Sowell, E. R. (2011). Imaging the impact of prenatal alcohol exposure on the structure of the developing human brain. Neuropsychol. Rev. 21, 102-118. doi: 10.1007/s11065-011-9163-0

Longo, A. C., Fried, A. P., Cameron, I., and Smith, A. M. (2014). The long-term effects of prenatal nicotine exposure on verbal working memory: an fMRI study of young adults. Drug Alcohol Depend. 144, 61-69. doi: 10.1016/j.drugalcdep. 2014.08.006

May, P. A., Brooke, L., Gossage, J. P., Croxford, J., Adnams, C., Jones, K. L., et al. (2000). Epidemiology of fetal alcohol syndrome in a South African community in the Western Cape Province. Am. J. Public Health 90, 1905-1912. doi: 10.2105/ajph.90.12.1905

May, P. A., Marais, A. S., De Vries, M. M., Kalberg, W. O., Buckley, D., Hasken, J. M., et al. (2016). The continuum of fetal alcohol spectrum disorders in a community in South Africa: Prevalence and characteristics in a fifth sample. Drug Alcohol Depend. 168, 274-286. doi: 10.1016/j.drugalcdep.2016. 09.025

May, P. A., Tabachnick, B. G., Gossage, J. P., Kalberg, W. O., Marais, A. S., Robinson, L. K., et al. (2013). Maternal factors predicting cognitive and behavioral characteristics of children with fetal alcohol spectrum disorders. J. Dev. Behav. Pediatr. 34, 314-325. doi: 10.1097/DBP.0b013e31829 05587

Meintjes, E. M., Jacobson, J. L., Molteno, C. D., Gatenby, J. C., Warton, C., Cannistraci, C. J., et al. (2010). An fMRI study of number processing in children with fetal alcohol syndrome. Alcohol Clin. Exp. Res. 34, 1450-1464. doi: 10.1111/j.1530-0277.2010.01230.x

Meintjes, E. M., Narr, K. L., Van Der Kouwe, A. J., Molteno, C. D., Pirnia, T., Gutman, B., et al. (2014). A tensor-based morphometry analysis of regional differences in brain volume in relation to prenatal alcohol exposure. Neuroimage Clin. 5, 152-160. doi: 10.1016/j.nicl.2014.04.001

Menon, V., Rivera, S. M., White, C. D., Glover, G. H., and Reiss, A. L. (2000). Dissociating prefrontal and parietal cortex activation during arithmetic processing. NeuroImage 12, 357-365. doi: 10.1006/nimg. 2000.0613

Migliorini, R., Moore, E. M., Glass, L., Infante, M. A., Tapert, S. F., Jones, K. L., et al. (2015). Anterior cingulate cortex surface area relates to behavioral inhibition in adolescents with and without heavy prenatal alcohol exposure. Behav. Brain Res. 292, 26-35. doi: 10.1016/j.bbr.2015.05.037

Naccache, L., and Dehaene, S. (2001). The priming method: imaging unconscious repetition priming reveals an abstract representation of number in the parietal lobes. Cereb. Cortex 11, 966-974. doi: 10.1093/cercor/11.10.966

Piazza, M., Giacomini, E., Le Bihan, D., and Dehaene, S. (2003). Single-trial classification of parallel pre-attentive and serial attentive processes using functional magnetic resonance imaging. Proc. Biol. Sci. 270, 1237-1245. doi: 10.1098/rspb.2003.2356

Pinel, P., Dehaene, S., Riviere, D., and LeBihan, D. (2001). Modulation of parietal activation by semantic distance in a number comparison task. NeuroImage 14, 1013-1026. doi: 10.1006/nimg.2001.0913

Rajaprakash, M., Chakravarty, M. M., Lerch, J. P., and Rovet, J. (2014). Cortical morphology in children with alcohol-related neurodevelopmental disorder. Brain Behav. 4, 41-50. doi: 10.1002/brb3.191

Rubin, M., and Safdieh, J. E. (2016). Netter's Concise Neuroanatomy Updated Edition E-Book. Philadelphia, PA: Elsevier Health Sciences.

Sattler, J. M. (1992). Assessment of Children, Third Edition. San Diego, CA: Sattler Publisher, Inc.

Sobel, M. E. (1982). Asymptotic confidence intervals for indirect effects in structural equation models. Sociol. Methodol. 13, 290-312. doi: 10.2307/ 270723

Sowell, E. R., Thompson, P. M., Mattson, S. N., Tessner, K. D., Jernigan, T. L., Riley, E. P., et al. (2001). Voxel-based morphometric analyses of the brain in children and adolescents prenatally exposed to alcohol. Neuroreport 12, 515-523. doi: 10.1097/00001756-200103050-00018

Sowell, E. R., Thompson, P. M., Peterson, B. S., Mattson, S. N., Welcome, S. E., Henkenius, A. L., et al. (2002). Mapping cortical gray matter asymmetry patterns in adolescents with heavy prenatal alcohol exposure. NeuroImage 17, 1807-1819. doi: 10.1006/nimg.2002.1328

Streissguth, A. P., Barr, H. M., Olson, H. C., Sampson, P. D., Bookstein, F. L., and Burgess, D. M. (1994). Drinking during pregnancy decreases word attack and arithmetic scores on standardized tests: adolescent data from 
a population-based prospective study. Alcohol Clin. Exp. Res. 18, 248-254. doi: 10.1111/j.1530-0277.1994.tb00009.x

Streissguth, A. P., Bookstein, F. L., Sampson, P. D., and Barr, H. M. (1989). Neurobehavioral effects of prenatal alcohol: Part III. PLS analyses of neuropsychologic tests. Neurotoxicol. Teratol. 11, 493-507. doi: 10.1016/08920362(89)90026-3

Tisdall, M. D., Hess, A. T., Reuter, M., Meintjes, E. M., Fischl, B., and van der Kouwe, A. J. (2012). Volumetric navigators for prospective motion correction and selective reacquisition in neuroanatomical MRI. Magn. Reson. Med. 68, 389-399. doi: 10.1002/mrm.23228

Van Der Kouwe, A. J. W., Benner, T., Salat, D. H., and Fischl, B. (2008). Brain morphometry with multiecho MPRAGE. NeuroImage 40, 559-569. doi: 10.1016/j.neuroimage.2007.12.025

Watkins, K. E., Paus, T., Lerch, J. P., Zijdenbos, A., Collins, D. L., Neelin, P., et al. (2001). Structural asymmetries in the human brain: a voxel-based statistical analysis of 142 MRI scans. Cereb. Cortex 11, 868-877. doi: 10.1093/cercor/ 11.9 .868

Winer, B. J. (1971). Statistical Principles in Experimental Design. New York, NY: McGraw-Hill.

Woods, K. J., Jacobson, S. W., Molteno, C. D., Jacobson, J. L., and Meintjes, E. M. (2017). Altered parietal activation during non-symbolic number comparison in children with prenatal alcohol exposure. Front. Hum. Neurosci. 11:627. doi: $10.3389 /$ fnhum.2017.00627
Woods, K. J., Meintjes, E. M., Molteno, C. D., Jacobson, S. W., and Jacobson, J. L. (2015). Parietal dysfunction during number processing in children with fetal alcohol spectrum disorders. NeuroImage Clin. 8, 594-605. doi: 10.1016/j.nicl. 2015.03.023

Woods, R. P. (2003). Multitracer: a java-based tool for anatomic delineation of greyscale volumetric images. NeuroImage 19, 1829-1834. doi: 10.1016/s10538119(03)00243-x

Zlatkina, V., and Petrides, M. (2014). Morphological patterns of the intraparietal sulcus and the anterior intermediate parietal sulcus of Jensen in the human brain. Proc. Biol. Sci. 281:20141493. doi: 10.1098/rspb. 2014.1493

Conflict of Interest: The authors declare that the research was conducted in the absence of any commercial or financial relationships that could be construed as a potential conflict of interest.

Copyright (c) 2021 Miles, Warton, Meintjes, Molteno, Jacobson, Jacobson and Warton. This is an open-access article distributed under the terms of the Creative Commons Attribution License (CC BY). The use, distribution or reproduction in other forums is permitted, provided the original author(s) and the copyright owner(s) are credited and that the original publication in this journal is cited, in accordance with accepted academic practice. No use, distribution or reproduction is permitted which does not comply with these terms. 